\title{
Slow nucleation rates in Chain Inflation with QCD Axions or Monodromy
}

\author{
Amjad Ashoorioon * Katherine Freese ${ }^{7}$ and James T. Liu \\ Michigan Center for Theoretical Physics, University of Michigan, Ann Arbor, Michigan 48109-1040, USA
}

(Dated: August 21, 2018)

\begin{abstract}
The previous proposal (by two of us) of chain inflation with the QCD axion is shown to fail. The proposal involved a series of fast tunneling events, yet here it is shown that tunneling is too slow. We calculate the bubble nucleation rates for phase transitions in the thick wall limit, approximating the barrier by a triangle. A similar problem arises in realization of chain inflation in the string landscape that uses series of minima along the monodromy staircase around the conifold point. The basic problem is that the minima of the potential are too far apart to allow rapid enough tunneling in these two models. We entertain the possibility of overcoming this problem by modifying the gravity sector to a Brans-Dicke theory. However, one would need extremely small values for the Brans-Dicke parameter. Many successful alternatives exist, including other "axions" (with mass scales not set by QCD) or potentials with comparable heights and widths that do not suffer from the problem of slow tunneling and provide successful candidates for chain inflation.
\end{abstract}

PACS numbers:

\section{INTRODUCTION}

In the old picture of inflation, originally suggested by A. Guth [1], the universe is trapped in a meta-stable false vacuum whose energy causes the universe to expand quasi-exponentially. As the universe expands, bubbles of true vacuum gradually form inside the sea of false vacuum. The transition is completed by the coalescence of bubbles, releasing the latent heat of transition and recovering from the supercooling. To have the universe expand sufficiently to solve the problems of standard big bang cosmology, the phase transition should be quite slow. This would prevent the bubbles from percolation and thus the universe would never recover from the inflationary stage.

Chain inflation [2] resolves the problems of old inflation by assuming that more than one stage of inflation is responsible for solving the problems of Big Bang cosmology. Instead, the universe tunnels rapidly through a series of ever lower energy vacua. The failure of old inflation is avoided because the bubbles of true vacuum are able to percolate at each step since the phase transition is fairly rapid. The nucleation rate per unit four volume has the form [3]:

$$
\Gamma=A e^{-S_{E}}
$$

where $S_{E}$ is the Euclidean action for the bounce solution extrapolating between false and true vacua and $A$ is a determinant factor [4], which is generally of order the quartic power of the energy scale of the phase transition. For a first order phase transition, with Einstein gravity, it has been shown that the probability of a point remaining

\footnotetext{
*Electronic address: amjad@umich.edu

${ }^{\dagger}$ Electronic address: ktfreese@umich.edu

${ }^{\ddagger}$ Electronic address: jimliu@umich.edu
}

in the false vacuum is given by

$$
p(t) \sim \exp \left(-\frac{4 \pi}{3} \beta H t\right),
$$

where $\beta$ is defined by

$$
\beta=\frac{\Gamma}{H^{4}} .
$$

Writing Eq. (I.2) as $\exp (-t / \tau)$, the lifetime of the field in the false vacuum is given by:

$$
\tau=\frac{3}{4 \pi} \frac{H^{3}}{\Gamma}
$$

The number of $e$-foldings for the tunneling event is

$$
\chi=\int H d t \sim H \tau=\frac{3}{4 \pi} \frac{H^{4}}{\Gamma} .
$$

$\beta$ has to be greater than the critical value, $\beta_{c}$, where [6] 34]

$$
\beta_{c}=9 / 4 \pi,
$$

to achieve percolation and thermalization. This corresponds to having an upper bound on the number of $e$ foldings that that can be obtained in each stage of inflation:

$$
\chi \leq \chi_{c}=\frac{1}{3} .
$$

The approximate number of $e$-foldings which is required to solve the problems of standard cosmology depends on the energy scale of inflation, $M$, and the reheating temperature:

$$
N_{e}=45+\ln (3000)+\frac{2}{3} \ln \left(M_{14}\right)+\frac{1}{3} \ln \left(T_{10}\right),
$$

where $M=M_{14} 10^{14} \mathrm{GeV}, T_{R H}=T_{10} 10^{10} \mathrm{GeV}$. For a model with energy scale and reheating temperature 
around the GUT scale, about $60 e$-foldings are required, whereas for the one in which these parameters take values around the QCD scale, minimum number of $e$-foldings is reduced to 25 . This corresponds to having at least around 200 phase transitions for the GUT scale chain inflation and around 100 ones for the QCD scale chain inflation.

A study of density perturbations from chain inflation has been performed in 7, 8], which found that the right amount of perturbations to match data can be generated. Previous studies can be found in [9, 10, 11].

Chain inflation can arise in many contexts. However, we wish to make clear at the outset that many other versions of chain inflation may be very successful. For example, Freese, Liu, and Spolyar [12] examined chain inflation due to four-forms in string theory where there can be large numbers of potential minima connected by tunneling. We note that the present result does not apply to the case of four-form inflation.

In this paper we focus specifically on two variants of chain inflation and show that the model fails for these cases: i) chain inflation with the QCD axion [13] and ii) monodromy chain inflation [7, 14]. The basic problem is that, for these two specific examples, the width of the potential is so large that tunneling never takes place. Individual minima are (meta)stable for extremely long timescales. We use a triangle potential to approximate the real potentials in obtaining these results. However, if one considers "axions" at any scale, not tied to QCD, then chain inflation can work for the scales discussed here in the paper. In addition, for potentials with comparable heights and widths, the problem can be avoided as well. Chain inflation fails for some select models but can easily work in others.

First we will review QCD axion inflation and remind the reader how the necessity to avoid the thin wall limit arose in the original paper [13]. Then we calculate the nucleation rate for the thick wall limit, approximating the potential with triangles. Next we will relax the restriction of taking the inflaton to be the QCD axion. If the inflaton is some other axion with different mass scales, we can set some constraints on the height and width of the bumps that separate two adjacent minima, in order to have critical nucleation rates. These constraints could be used in the future to identify proper candidates for realizing chain inflation. Finally, inspired by extended inflation [16], we investigate the possibility of overcoming the difficulties of QCD axion inflation by modifying the gravity sector of the theory.

\section{CHAIN INFLATION WITH THE QCD AXION}

Two of us [13] previously proposed that the QCD axion might be the inflaton if it were supplemented with i) a large number of minima, corresponding to new heavy fermions participating in the chiral anomaly and ii) soft symmetry breaking of the Peccei-Quinn symmetry to provide a tilt to the potential. Here we discuss this model in detail and examine its pitfalls as a proposed inflation model.

The QCD axion is the Goldstone boson of the broken $U(1)_{\mathrm{PQ}}$ Peccei-Quinn symmetry which was postulated to solve the strong $C P$ problem of strong interactions [17, 18]. As was shown by Sikivie [19], when this global symmetry is broken, a discrete $\mathbb{Z}_{N}$ subgroup remains unbroken, where $N$ is the number of quark flavors that rotate under $U(1)_{\mathrm{PQ}}$. The complete form of the axion potential depends on non-perturbative effects. However, for definiteness, we will focus on the "invisible axion" model of Dine-Fischler-Srednicki-Zhitnitsky [20, 21], where the axion is identified as the phase of a complex $S U(2) \times U(1)$ singlet scalar, $\sigma=\frac{v+\rho}{\sqrt{2}} \exp (i a / v)$. Below the Peccei-Quinn symmetry breaking scale, $v / \sqrt{2}$, the axion potential takes the following form:

$$
V(a)=V_{0}\left[1-\cos \left(\frac{N a}{v}\right)\right] .
$$

Here $v=f_{a} N$, where $f_{a}$ is the decay constant of the axion; it has to be between $10^{9} \mathrm{GeV}$ and $10^{12} \mathrm{GeV}$ to satisfy the constraints from stellar evolution [22, 23] and cosmology [24]. In addition, $V_{0}=m_{a}^{2} f_{a}^{2}$ is on the order of the QCD scale, which we take to be around 220 $\mathrm{MeV}$. The axion mass, $m_{a}$, is equal to $2 N \frac{\sqrt{z}}{1+z} f_{\pi} m_{\pi} / v$, where $z=m_{u} / m_{d}=0.56$ (but see [25]), and $m_{\pi}=135$ $\mathrm{MeV}$ and $f_{\pi}=93 \mathrm{MeV}$ are respectively the mass and decay constant of the pion. The energy scale of inflation is around the QCD scale, and thus, as pointed out above, the required number of minima has to be taken to be larger than 75 to obtain a sufficient number of $e$-foldings. This requires the introduction of additional heavy fermions beyond the usual quark and leptons. They carry color charge as well as PQ charge and thus contribute to the QCD anomaly [19]. To be definite hereafter we take $N=100$.

The $U(1)_{\mathrm{PQ}}$ symmetry is softly broken by adding a term of the form $\mu^{3} \sigma+$ h.c. to the Lagrangian. This in turn adds a term of the form $\eta \cos (a / v+\gamma)$ to the axion potential, where $\eta$ and $\gamma$ are real parameters. (Note that $\gamma$ misaligns the QCD and soft breaking minima.) Introducing a new variable $\theta \equiv a / f_{a}$, the combined potential for the axion takes the form:

$$
V(\theta)=V_{0}(1-\cos \theta)-\eta \cos \left(\frac{\theta}{N}+\gamma\right)
$$

There is a limit on $\gamma$ from the electric dipole moment (EDM) of the neutron, namely, from the fact that the minimum of the combined potential should not be shifted from zero:

$$
\left.\Delta \theta\right|_{\mathrm{EDM}}<6 \times 10^{-10} .
$$

We should impose the EDM bounds at the bottom of the potential, which is the end point of tunneling. There one can show that $\Delta \theta=\left|\frac{\eta \sin \gamma}{V_{0} N}\right|[13]$. This constraint can be 


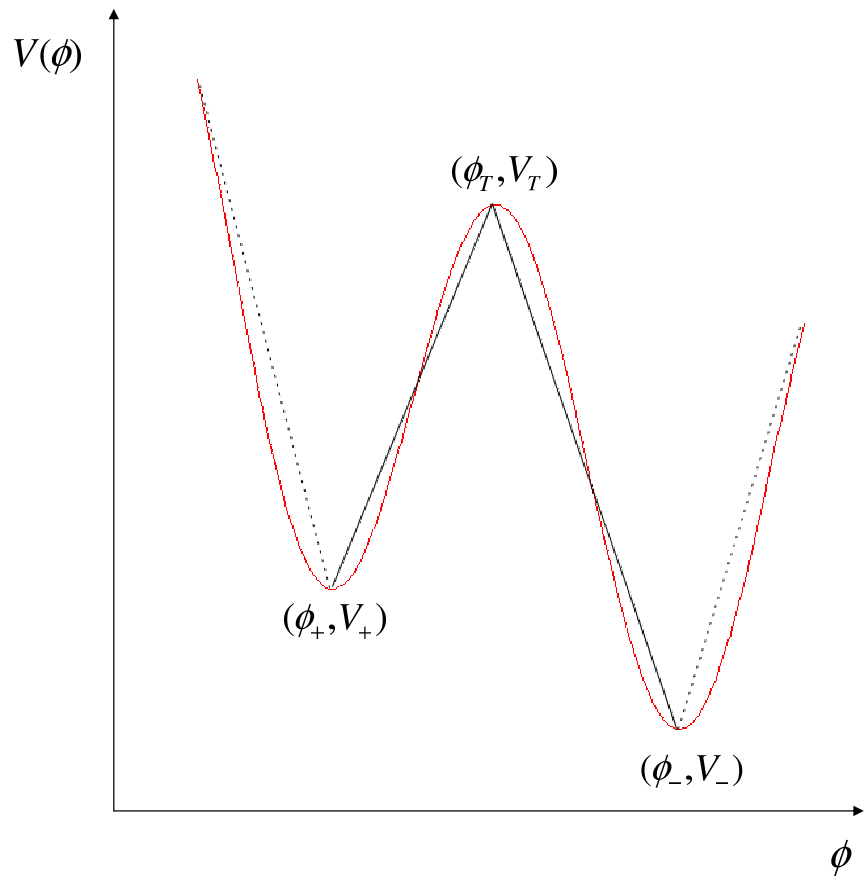

FIG. 1: To calculate the nucleation rate we have approximated the potential with a triangle.

satisfied in many ways as was discussed in [13]. Here, for simplicity, we set $\gamma=0$ from the outset.

In the thin wall limit, the Euclidean action is given by

$$
S_{E}=\frac{27 \pi^{2} S_{1}^{2}}{2 \epsilon^{3}}
$$

where $S_{1}$ is given by

$$
S_{1}=\int_{0}^{2 \pi f_{a}} V_{0}\left[1-\cos \left(\frac{a}{f_{a}}\right)\right] d a .
$$

Thus, we have:

$$
S_{E}=5 \times 10^{5} \frac{V_{0}^{2} f_{a}^{4}}{\epsilon^{3}}
$$

For the parameters of the invisible axion [20], $S_{E} \gg 1$ and thus tunneling is suppressed. In [13], it was speculated that being away from the thin wall limit and having $\epsilon \lesssim V_{0}$, can cure this problem. However as we will show below, the problem of suppressed tunneling persists even in the thick wall limit.

\section{THICK WALL LIMIT}

To compute the nucleation rate, we will use the result of [26], where a general one dimensional potential could be approximated by a potential with triangular form (see Fig. 1), in which case the equation of motion for the bounce can be explicitly solved. Note that $\left(\phi_{+}, V_{+}\right)$and $\left(\phi_{-}, V_{-}\right)$are the values of the field and potential energies at the false and true vacua, respectively, and $\left(\phi_{T}, V_{T}\right)$ is the value of the field and potential energy at the maximum of the potential. The resulting expression for the Euclidean action takes on two forms, depending on the condition [35]:

$$
\left(\frac{\Delta V_{-}}{\Delta V_{+}}\right)^{1 / 2} \geq \frac{2 \Delta \phi_{-}}{\Delta \phi_{-}-\Delta \phi_{+}}
$$

Here we have defined the quantities $\Delta \phi_{ \pm} \equiv \pm\left(\phi_{T}-\phi_{ \pm}\right)$, $\Delta V_{ \pm} \equiv\left(V_{T}-V_{ \pm}\right)$and $\lambda_{ \pm} \equiv \Delta V_{ \pm} / \Delta \phi_{ \pm}$. If the above condition is satisfied, $S_{E}$ takes the form:

$$
S_{E}=\frac{32 \pi^{2}}{3} \frac{1+c}{(\sqrt{1+c}-1)^{4}}\left(\frac{\Delta \phi_{+}^{4}}{\Delta V_{+}}\right)
$$

where $c \equiv \lambda_{-} / \lambda_{+}$. Otherwise, if condition (III.1) is not met, the Euclidean action is instead given by:

$$
S_{E}=\frac{1}{96} \pi^{2} \lambda_{+}{ }^{2} R_{T}^{2}\left(-\beta_{+}^{3}+3 c \beta_{+}^{2} \beta_{-}+3 c \beta_{+} \beta_{-}^{2}-c^{2} \beta_{-}^{3}\right),
$$

where:

$$
\beta_{ \pm} \equiv \sqrt{\frac{8 \lambda \phi_{ \pm}}{\lambda_{ \pm}}}, \quad R_{T} \equiv \frac{1}{2}\left(\frac{\beta_{+}^{2}+c \beta_{-}^{2}}{c \beta_{-}-\beta_{+}}\right) .
$$

In order to connect the parameters of the axion potential to that of the triangle approximation, we first note that $\epsilon$, the energy difference between two consecutive minima, is proportional to the soft-breaking parameter $\eta$. However we cannot make $\eta$ arbitrarily large because once $\eta$ becomes equal to $N V_{0}$ the barrier between two vacua disappears and the field simply rolls down the potential rather than tunneling. Thus, if $N$ happens to be close to the critical value, $N_{c} \approx 100$, for which one would obtain enough $e$-foldings, the model will not be able to inflate enough if $\eta$ is larger that $N_{c} V_{0}$. Hereafter, we will take $\eta=\left(N_{c}-1\right) V_{0}$ since it gives the most rapid tunneling rate without going over to rolling.

We note that, in the middle of the axion potential, the condition (III.1) is satisfied and hence we may use Eq. (III.2) to compute the instanton action. For $f_{a}=$ $10^{12} \mathrm{GeV}$ (or $10^{9} \mathrm{GeV}$ ), we see that $S_{E}$ is of order $10^{46}$ (or $10^{34}$ ) in the middle of the potential. Thus the tunneling is highly suppressed. At the bottom of the potential, where $\epsilon$ gets smaller, condition (III.1) is not met and we have to use equation (III.3) to calculate the Euclidean action. Here the situation gets worse; for $f_{a}=10^{12} \mathrm{GeV}$ (or $10^{9} \mathrm{GeV}$ ), the Euclidean action takes the value $10^{53}$ (or $10^{41}$ ). The minute value of the nucleation rate can be understood intuitively by noting that the vacua are very far apart and hence their wave functions cannot overlap.

Somewhere in the limit of a broad barrier, the Coleman transition [3] ceases to exist. Then the decay instead goes through the Hawking-Moss instanton [27], which physically describes a thermal jump of the field onto the top of the barrier. However, to have Hawking-Moss instanton, 
we must have $0 \leqslant m^{2} \leqslant 2 H^{2}$. This condition is not satisfied in the case of the QCD axion, for which $m_{a}^{2} \sim V_{0} / f_{a}^{2}$, as $f_{a} \ll M_{\mathrm{Pl}}$. Thus we conclude that the QCD axion, as suggested in 13], is not able to realize chain inflation.

"Axions" not tied to the QCD scale are also ubiquitous in string theory [28]. If we relax the requirement that we are dealing with the QCD axion here, and consider "axions" of arbitrary mass scale, successful chain inflation models can be found that avoid the problem of insufficient tunneling. For a potential of the form (II.2) with arbitrary barrier height $V_{0}$ and decay constant $f_{a}$, the instanton action $S_{E}$ can become of $\mathcal{O}(1)$, if the following constraint is satisfied:

$$
f_{a} \lesssim 10^{-2} V_{0}^{1 / 4}
$$

It would be worthwhile to investigate the realm of string theory axions to see if one can find suitable candidates that satisfy (III.5) to realize the idea of chain inflation.

A similar problem of suppressed nucleation rates arises in a particular attempt to realize chain inflation in the string landscape [7]. In [15], the generic distance between minima in the monodromy staircase is obtained to be large, $\mathcal{O}\left(M_{\mathrm{Pl}}\right)$. As 7] requires a shorter inter-minimum distance, this will again lead to suppressed nucleation rates.

\section{EXTENDING CHAIN INFLATION}

In the remainder of this paper, we suggest a way of avoiding the problem of suppressed tunneling rates of QCD and monodromy chain inflation by modifying the gravity sector of the theory. The remedy is motivated by how extended inflation [16] amends the graceful exit problem of old inflation.

Several approaches have been taken to solve the graceful exit problem of old inflation. Adams and Freese 29] suggested a time-dependent nucleation rate, so that the initial value of $\beta$ defined in Eq. (I.3) was very small (and the universe inflates) but the final value of $\beta$ becomes very large (so that the bubbles of true vacuum percolate and the phase transition completes); their model is called double field inflation.

Around the same time, La and Steinhardt [16] made an alternative suggestion, namely extended inflation, where the gravity part of the action takes a Brans-Dicke (BD) form [30],

$$
\mathcal{L}_{\mathrm{BD}}=\frac{1}{2} \int \sqrt{-g} d^{4} x\left[-\Phi R+\omega\left(\frac{\partial^{\mu} \Phi \partial_{\mu} \Phi}{\Phi}\right)\right],
$$

where $\omega$ is known as the Brans-Dicke parameter. In the above action, general relativity is recovered in the limit of $\omega \rightarrow \infty$ and present day tests of general relativity require $\omega>500$. Modification of the gravity sector as above will change the expansion from de-Sitter to [16]:

$$
a(t)=\left(1+\frac{\chi t}{\alpha}\right)^{\omega+\frac{1}{2}}
$$

Here $\alpha^{2}=(3+2 \omega)(5+6 \omega) / 12$ and $\chi^{2} \equiv 8 \pi \rho_{0} / 3 \Phi(0)$, where $\Phi(0)$ is $m_{\mathrm{Pl}}^{2}$ in the beginning of inflation at $t=0$ and $\rho_{0}=V(0)$ is the value of cosmological constant in the metastable vacuum. For $t \lesssim t_{\mathrm{dS}}$, where $t_{\mathrm{dS}} \simeq \alpha / \chi$, the scale factor expands exponentially. However after that the behavior of the scale factor changes to power-law, $t^{q}$, where $q=\omega+1 / 2$. Change in the evolution of the background modifies the rate at which bubble nucleation converts false vacuum to true vacuum.

As shown in [5], the probability of a point remaining in the false vacuum is given by

$$
\left.p(t)=\exp \left[-\int^{t} d t^{\prime} \Gamma\left(t^{\prime}\right) a^{3}\left(t^{\prime}\right)\right) \frac{4 \pi}{3}\left(\int_{t^{\prime}}^{t} \frac{t^{\prime \prime}}{a\left(t^{\prime \prime}\right)}\right)^{3}\right] .
$$

For de-Sitter expansion, this reduces to (I.2). However, for a power-law background, the probability to remain in false vacuum phase becomes:

$$
p(t)=\exp \left(-\frac{\pi}{3} \beta \omega y^{4}\right),
$$

where $y \equiv \chi t / \omega[16]$. As $p(t)$ is decreasing much faster than the volume, the universe will soon exit inflation and graceful exit is achieved. However, for the case of extended inflation 16], it was understood that the bubbles that form in the beginning of inflation would be stretched to cosmological scales by inflation. This constraint forced $\omega$ to be less than 20 . With this value of $\omega$, requiring the scalar spectral index to satisfy constraints from WMAP is impossible. Resolutions that are based on making $\omega$ time-dependent are also very contrived [31]. Nonetheless, we may apply this idea to chain inflation to see if we can obtain a sensible, even though contrived, solution for the small nucleation rate of QCD and monodromy chain inflation.

We first calculate $t_{p}$, the time it takes for the universe to complete the power-law phase. The volume in the false vacuum phase is

$$
V_{f}=t^{3 q} p(t),
$$

where $p(t)$ is given in Eq. (IV.4). The volume in the false vacuum phase starts to contract at time

$$
t_{c}=\left(\frac{9 q \omega^{3}}{4 \pi \beta}\right)^{1 / 4} \chi^{-1} .
$$

At $t_{c}$, the exponential factor in $p(t)$ starts to takes over the behavior of $V_{f}$ and thereafter it starts to decrease exponentially, with an exponent equal to $-\left(t / t_{c}\right)^{4}$. Only within $t_{p} \simeq$ few $\times t_{c}$, the volume in the false vacuum phase becomes an infinitesimal fraction of the total volume. Therefore the coalescence of true vacuum bubbles and recovery from the false vacuum phase is achieved within few $\times t_{c}$. The number of $e$-foldings obtained in the power-law phase is then:

$$
N_{p} \simeq q \ln \left(1+\frac{t_{p}}{t_{\mathrm{dS}}}\right) \simeq \ln \left(\frac{9 q \omega^{3}}{4 \pi \beta \alpha^{4}}\right) .
$$


This should be compared with the number of $e$-foldings obtained in the de-Sitter stage:

$$
N_{\mathrm{dS}}=\alpha .
$$

Note that Brans-Dicke theories with $\omega \simeq 1$ arise naturally in compactifications of sting theory and other Kaluza-Klein theories [32]. For $\omega=1$, we have

$$
\begin{aligned}
N_{p} & \simeq S_{E} \\
N_{\mathrm{dS}} & \simeq 2.5
\end{aligned}
$$

where $S_{E}$ is the Euclidean action for the bounce solution which is obtained in the last section. For QCD inflation, at the bottom of the potential, $S_{E}$ was obtained to be of order $10^{53}$ for $f_{a} \simeq 10^{12} \mathrm{GeV}$. Therefore the number of $e$ foldings obtained in the power-law phase of the first minimum is much more than enough and can solve the problems of standard Big Bang cosmology by itself. There is no need for the other minima in chain inflation. One can circumvent the long power-law phase by adjusting the value of $\omega$ to be of order $\beta \simeq \exp \left(-S_{E} / 3\right) \simeq \exp \left(-10^{53}\right)$. For such values of $\omega$ the universe expands $\sim$ one $e$-fold in the de-Sitter stage and recovers from the power-law phase in a small fraction of an $e$-fold. Although such small values of $\omega$ are in disagreement with solar tests of general gravity, it may be possible to make the parameter $\omega$ field-dependent (similar to hyper-extended inflation [33]), so that it takes such small values during inflation but is then driven toward experimentally-viable values afterwards.

In summary, we have identified a problem of too slow nucleation rates in chain inflation in those models with minima separated by great distances in field space. The two cases we studied were chain inflation with the QCD axion [13] and monodromy [7] chain inflation. To obtain sufficiently rapid tunneling, one could look for other "axionic" candidates that satisfy constraints like (III.5). We have also suggested another option of resolving the problem by extending the gravity sector of the theory to Brans-Dicke theory. This solution is motivated by the mechanism whereby extended inflation solves the graceful exit problem of old inflation (which also arises due to the small nucleation rate of the inflaton). Such a resolution requires an extremely small value for the BransDicke parameter, $\omega$, which must therefore become time dependent. It is interesting to see if string theory can come up with such values for $\omega$. Alternatively, many models of chain inflation which have smaller distances between vacua (or are not described by a potential at all) easily have rapid tunneling and do not suffer from the problems discussed in this paper at all.

\section{Acknowledgments}

We are grateful to D. Spolyar, K. Bobkov and M. Soroush for useful discussions. A.A. is partially supported by NSERC of Canada. This work was supported in part by the US Department of Energy under grant DE-FG02-95ER40899 and the Michigan Center for Theoretical Physics.
[1] A. H. Guth, Phys. Rev. D 23, 347 (1981).

[2] K. Freese and D. Spolyar, JCAP 0507, 007 (2005) arXiv:hep-ph/0412145.

[3] S. R. Coleman, Phys. Rev. D 15, 2929 (1977) [Erratumibid. D 16, 1248 (1977)].

[4] C. G. Callan and S. R. Coleman, Phys. Rev. D 16, 1762 (1977).

[5] A. H. Guth and E. J. Weinberg, Nucl. Phys. B 212, 321 (1983).

[6] M. S. Turner, E. J. Weinberg and L. M. Widrow, Phys. Rev. D 46, 2384 (1992).

[7] D. Chialva and U. H. Danielsson, arXiv:0804.2846 [hepth].

[8] D. Chialva and U. H. Danielsson, arXiv:0809.2707 [hepth].

[9] S. Watson, M. J. Perry, G. L. Kane and F. C. Adams, JCAP 0711, 017 (2007) arXiv:hep-th/0610054.

[10] B. Feldstein and B. Tweedie, JCAP 0704, 020 (2007) arXiv:hep-ph/0611286.

[11] Q. G. Huang, JCAP 0705, 009 (2007) arXiv:0704.2835 [hep-th]].

[12] K. Freese, J. T. Liu and D. Spolyar, arXiv:hep-th/0612056

[13] K. Freese, J. T. Liu and D. Spolyar, Phys. Rev. D 72,
123521 (2005) arXiv:hep-ph/0502177.

[14] D. Chialva, U. H. Danielsson, N. Johansson, M. Larfors and M. Vonk, JHEP 0802, 016 (2008) arXiv:0710.0620 [hep-th]].

[15] M. C. Johnson and M. Larfors, arXiv:0805.3705 [hep-th].

[16] D. La and P. J. Steinhardt, Phys. Rev. Lett. 62, 376 (1989) [Erratum-ibid. 62, 1066 (1989)].

[17] R. D. Peccei and H. R. Quinn, Phys. Rev. Lett. 38, 1440 (1977).

[18] R. D. Peccei and H. R. Quinn, Phys. Rev. D 16, 1791 (1977).

[19] P. Sikivie, Phys. Rev. Lett. 48, 1156 (1982).

[20] M. Dine, W. Fischler and M. Srednicki, Phys. Lett. B 104, 199 (1981).

[21] A. R. Zhitnitsky, Sov. J. Nucl. Phys. 31, 260 (1980) [Yad. Fiz. 31, 497 (1980)].

[22] D. A. Dicus, E. W. Kolb, V. L. Teplitz and R. V. Wagoner, Phys. Rev. D 18, 1829 (1978).

[23] D. A. Dicus, E. W. Kolb, V. L. Teplitz and R. V. Wagoner, Phys. Rev. D 22, 839 (1980).

[24] L. F. Abbott and P. Sikivie, Phys. Lett. B 120, 133 (1983).

[25] M. R. Buckley and H. Murayama, arXiv:0705.0542 [hep$\mathrm{ph}$. 
[26] M. J. Duncan and L. G. Jensen, Phys. Lett. B 291, 109 (1992).

[27] S. W. Hawking and I. G. Moss, Phys. Lett. B 110, 35 (1982).

[28] P. Svrcek and E. Witten, JHEP 0606 (2006) 051 arXiv:hep-th/0605206.

[29] F. C. Adams and K. Freese, Phys. Rev. D 43, 353 (1991).

[30] C. Brans and R. H. Dicke, Phys. Rev. 124, 925 (1961).

[31] A. M. Green and A. R. Liddle, Phys. Rev. D 54, 2557 (1996) arXiv:astro-ph/9604001.

[32] P. G. O. Freund, Nucl. Phys. B 209, 146 (1982).
[33] P. J. Steinhardt and F. S. Accetta, Phys. Rev. Lett. 64, 2740 (1990).

[34] As was shown in [5], requiring bubble nucleation is different from termination of eternal inflation. The critical value for percolation $\beta$ lies somewhere between $1.1 \times 10^{-6}$ and $9 n_{c} / 4 \pi$, where $n_{c}=0.34$ is the critical ratio between the volume inside the bubbles and the total volume. However, we have been inexact and used the term bubble percolation as equivalent to the end of inflation.

[35] $\Delta \phi_{-}>\Delta \phi_{+}$is assumed to be satisfied already. 